\title{
VoIP Performance Analysis over IPv4 and IPv6
}

\author{
Monjur Ahmed, Alan T Litchfield, Shakil Ahmed \\ Auckland University of Technology, New Zealand. \\ E-mail: \{monjur.ahmed, alan.litchfield, shakil.ahmed\}@aut.ac.nz
}

\author{
Adnan Mahmood \\ Freelance IT consultant, Surrey, United Kingdom \\ E-mail: rdadnan@yahoo.com
}

Md. Emran Hossain Meazi

America Bangladesh University, Dhaka, Bangladesh

E-mail: emran_mba@ymail.com

\begin{abstract}
The advance of technology often requires the emergence of complementary technologies, of which the transition from IPv4 to IPv6 presents a significant example. The move of protocol has focussed attention on the level of performance for associated technologies. Among the many Internet applications, in contemporary digital communications, VoIP stands apart in importance. This paper presents a performance analysis of VoIP using IPv4 and IPv6. Using OPNET to simulate the protocols and to investigate areas of performance weakness.
\end{abstract}

Index Terms-IPv4, IPv6, Performance, VoIP, VoIPv4, VoIPv6.

\section{INTRODUCTION}

The need to introduce IPv6 has forced the computing world to scrutinize the new version of IP. Extensive research that relates to the transition from IPv4 to IPv6 has explored the behaviour of applications that use IPv6 $[1,2,3,4]$. While the transition from IPv4 to IPv6 is inevitable, the accommodation of the existing concept of 'Internet' and its associated applications must work in the new environment [1]. The replacement of IPv4 by IPv6 is inevitable and thus to is the suitability of applications for IPv6. This presents the need to study the performance of applications in IPv6 that are currently running on IPv4 [5] The significance of the study is twofold: it will help to determine the suitability of an existing application to be implemented under IPv6, and the degree of improvements required for an application to be compatible with IPv6 based communication networks. Given the significance of Voice over Internet Protocol (VoIP) [6, 7], it is important to consider the suitability of deployment of VoIP in IPv6 based networks [2, 8].

This paper takes a comparative approach to study the level of performance of VoIPv6 and VoIPv4. Under a simulated environment, Quality of Service (QoS) parameters are observed for VoIPv4 and VoIPv6 to establish the suitability and readiness of VoIP for IPv6 and the level at which VoIP be shows an impact from the transition.
The study presents an exploration of the performance trade-offs between IPv4 and IPv6. The paper is organized as follows: section 2 presents related work and defines the technologies. Section 3 outlines the OPNET simulation environment, network topology and configuration used in the study. OPNET is used as the simulation tool to carry out the test. The topology is then configured to form two scenarios: VoIPv4 and VoIPv6. Performance parameters are selected and used to compare the protocols. Section 4 presents the result of the simulation. In section 5, the collected statistics from the simulation are analysed to discover comparative performance trends between VoIPv4 and VoIPv6.The paper concludes with Section 6 which entails a critical analysis of the study, addressing significance of outcomes of the study as well as the possible limitations of the research carried out.

\section{RELATED WORKS}

Some studies claim that any single performance result cannot is not reliable for the determination of IP based network performance, that is there are a number of factors applicable to IPv4 and thus to VoIPv6 [9]. For example, audio codecs play an important role in the application of VoIP and so the performance of audio codecs and the level of coupling (high or low) have an impact on total quality and user experience [10].

Since some researchers doubt that IPv6 will deliver expected improvements over IPv4 [22] as the transition from IPv4 to IPVv6 occurs, performance of IPv6 based networks needs to be monitored. Nevertheless, IPv6 provides improved efficiency over IPv4 and therefore better QoS experiences [11]. The nature of VoIP in real time streaming means there is very little compromise on QoS and performance where VoIP is dependent upon a number of factors, ranging from protocols used, to type of network and medium used for the deployment of VoIP $[10,12]$. In addition, the underlying algorithm to process speech (or audio) in VoIP has a profound effect on QoS [13]. 
While some researchers suggest that VoIPv6 may have better performance for VoIP, performance fluctuations cannot be ignored. Additionally, the sophistication of the network infrastructure contributes to the overall quality of IPv6 based voice network deployment [2]. Packet delay variation and end-to-end packet delay has a profound effect on VoIP QoS [6] which makes it crucial to observe these parameters for IPv4 and IPv6, for example Mean Opinion Score (MOS) and jitter [14, 15, 16, 7]. Security is also important [17], in relation to security features available for IP4 and IPv6. Security features are richer for IPv6 than IPv4 and this provides greater credibility for the protocol plus flexibility, lower maintenance overheads and extendibility $[18,19]$.

However, while IPv6 gives greater flexibility and assurance for security if deployed and configured correctly [20], the difference with IPv4 is not significant because both protocols are network layer based and are vulnerable to similar types of attack [19]. Studies show that IPv6 based VoIP deployment can deliver no less quality than IPv4 based VoIP deployment [21].

\section{SimUlation AND TEST}

The study is a simulation where OPNET is used as the simulation tool. Two network scenarios are developed with exactly same configuration and elements except for the IP address. One of the scenarios has been configured with IPv4 and the other with IPv6. Fig. 1 shows the network topology used in the study.

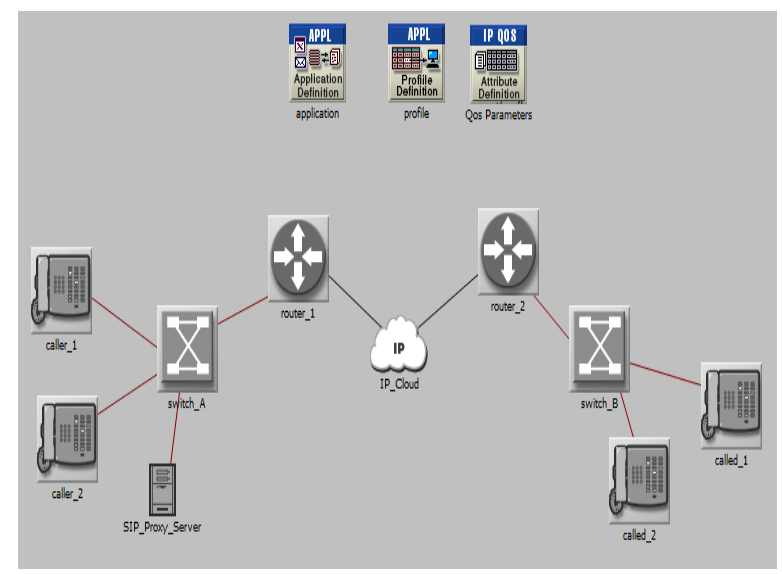

Fig 1. Network Topology

Two remote networks are connected through a public network infrastructure or cloud where the caller and called reside in remote networks. The inclusion of the cloud reflects a real life network and how the network will span geographically dispersed public network infrastructures. A SIP (Session Initiation Protocol) proxy server is used as the source of the signalling protocol. The major technical configuration for both the scenarios (IPv4 and IPv6 based) is summarized in table 1.

Figure 2 illustrates that full mesh traffic flows between all nodes has been configured for VoIP traffic with 1000 calls per hour.
Table 1. Technical Attributes and Values Used

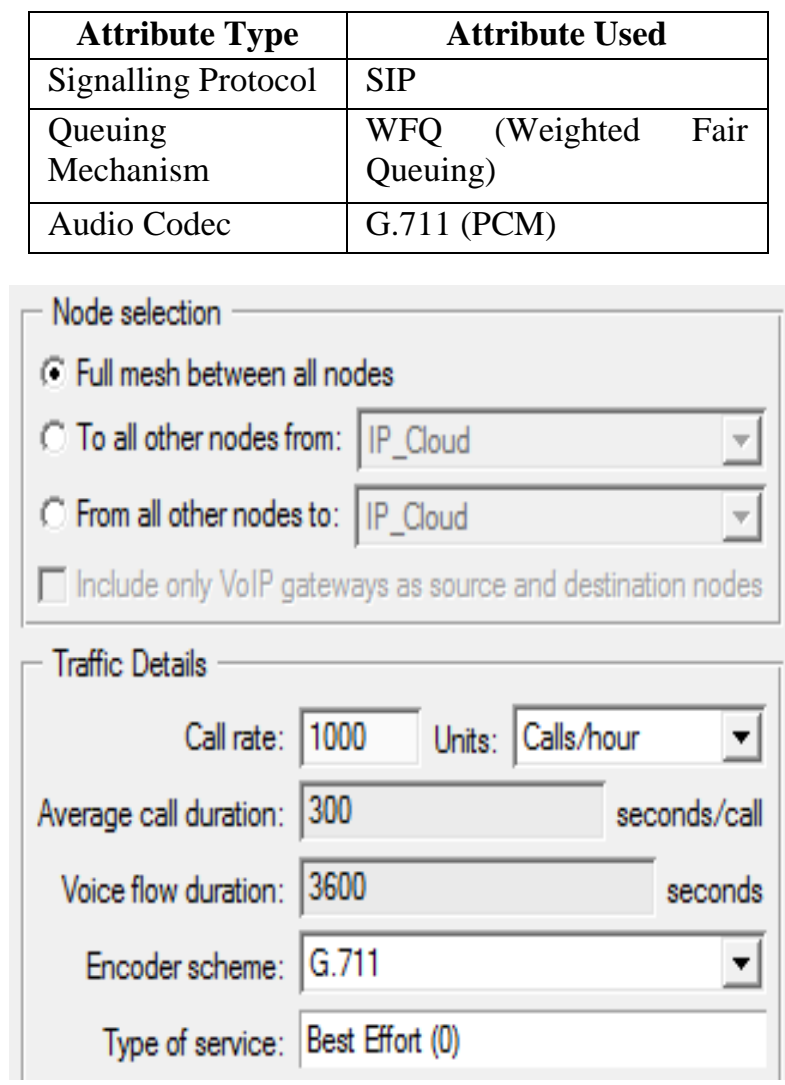

Fig 2. VoIP Traffic Flow Configuration

\section{RESULTS}

The results are derived from statistics about certain performance and QoS related parameters that have been carefully selected. Governing factors that directly and significantly relate to the performance of VoIP networks are traffic delay, traffic dropped, jitter, MOS, packet delay variation and End-to-End packet delay [12, 14, 16]. Figure 3 shows the trends of background IP traffic delay for VoIPv4 and VoIPv6 and the former has significantly higher background IP traffic delay. IP traffic drop is higher for IPv4, as illustrated in Figure 4, and gradually increases as time progresses from the point of network convergence.

The results from the simulation are combined to make it easier to compare outcomes for both the scenarios, VoIPv4 and VoIPv6. For any given parameter observed and statistics collected, the resultant trend is observed by means of graph for both VoIPv4 and VoIPv6 scenarios, as apparent from the illustrations of results obtained.

Jitter is one of the most important parameters to determine performance and QoS. Jitter for VoIPv4 and VoIPv6 are illustrated in Figure 5. Jitter is an undesirable phenomenon with respect to quality and performance. The study shows the jitter for VoIPv6 is close to zero or very insignificant while for VoIPv4, consistently demonstrates jitter and random fluctuations that increase in frequency gradually. 


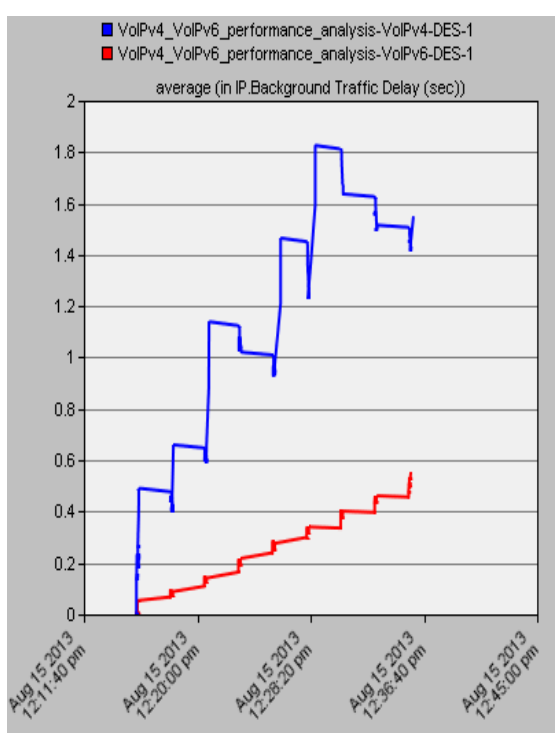

Figure 3. IP Background Traffic Delay

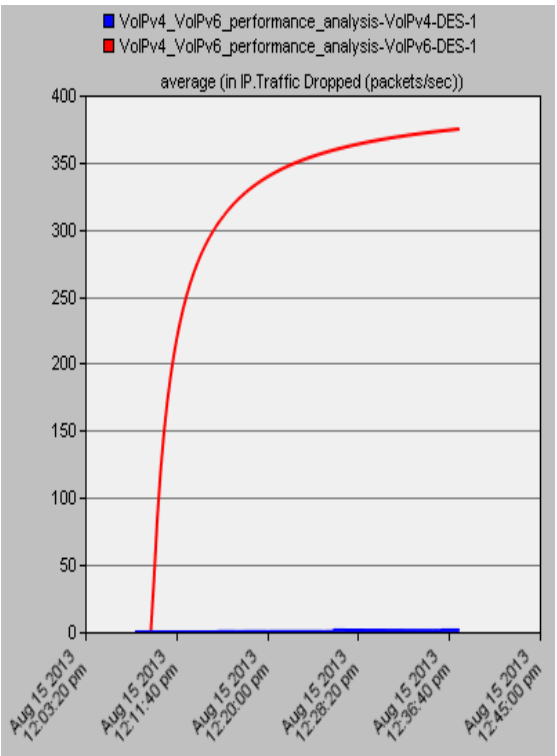

Figure 4. IP Traffic Dropped

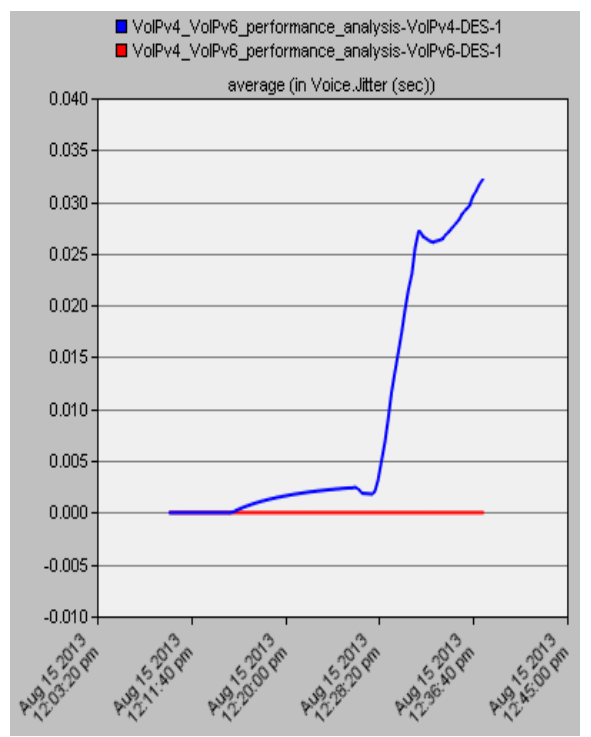

Figure 5. Jitter

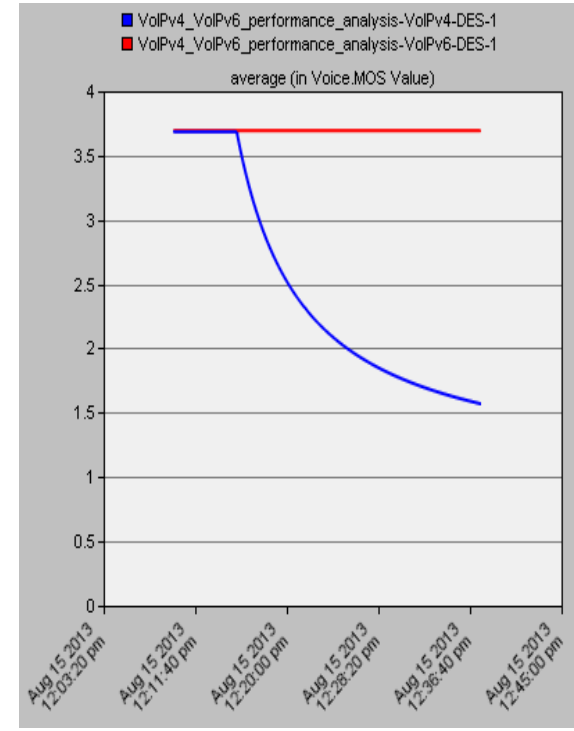

Figure 6. MOS Value

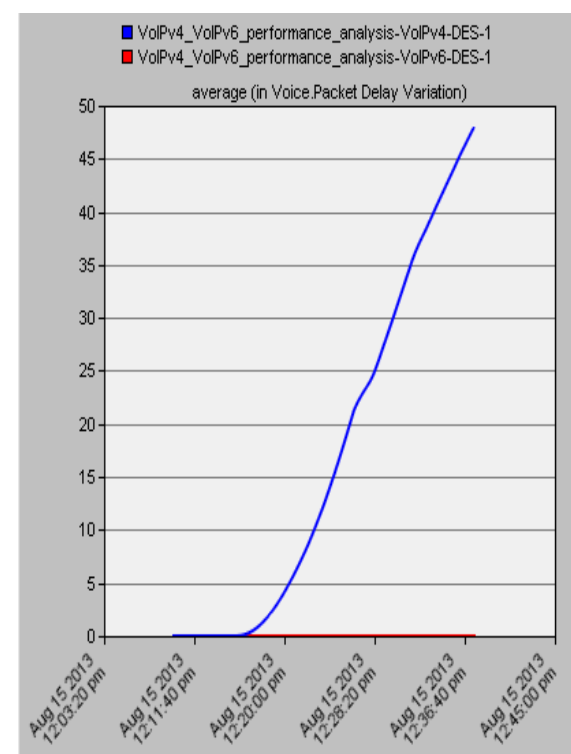

Figure 7. Packet Delay Variation

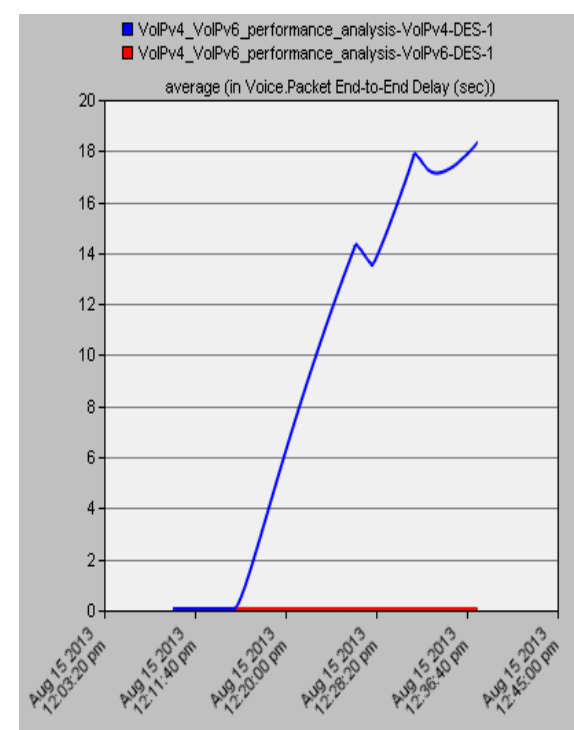

Figure 8. End-to-End Packet Delay 


\section{ANALYSIS OF RESULTS}

The scale for MOS is defined with a scale of 1 to 5 where 1 is the least efficient and 5 the most efficient performance indicator for a communication network [16]. The MOS values observed for the study are shown in Figure 6 and illustrates consistency in MOS values for VoIPv6 of around 3.7. As time progresses in a live communication scenario with on-going communication instances or voice calls for VoIP networks, the IPv4 based deployment gradually decreases MOS values from 3.7 to 1.6 (Figure 6).

VoIPv4 displays higher packet delay with significant increases in variation (Figure 7) while VoIPv6 shows no performance issues. Conceptually similar characteristics are shown in Figure 8 for End-to-End packet delay variation. Note how the results between Figures 7 and 8 are similar.

Table 2 and table 3 summarize the findings obtained from the simulation. They represent QoS and delay parameters.

Table 2. Comparison of QoS parameters

\begin{tabular}{|c|c|c|c|}
\hline & MOS & Jitter & $\begin{array}{c}\text { IP Traffic } \\
\text { Dropped }\end{array}$ \\
\hline VoIPv4 & $\begin{array}{c}3.7-1.6 \\
\text { (Gradually } \\
\text { decreasing) }\end{array}$ & $\begin{array}{c}\text { Gradually } \\
\text { increasing }\end{array}$ & $\begin{array}{c}\text { Gradually } \\
\text { increasing }\end{array}$ \\
\hline VoIPv6 & 3.7 & Insignificant & Very low \\
\hline
\end{tabular}

Table 3. Comparison of QoS Delay Parameters

\begin{tabular}{|c|c|c|c|}
\hline & $\begin{array}{c}\text { End-to- } \\
\text { End Packet } \\
\text { Delay }\end{array}$ & $\begin{array}{c}\text { Packet } \\
\text { Delay } \\
\text { Variation }\end{array}$ & $\begin{array}{c}\text { Background IP } \\
\text { Traffic Delay }\end{array}$ \\
\hline $\begin{array}{c}\text { VoIP } \\
\text { v4 }\end{array}$ & $\begin{array}{c}\text { Gradually } \\
\text { increasing }\end{array}$ & $\begin{array}{c}\text { Gradually } \\
\text { increasing }\end{array}$ & $\begin{array}{c}\text { Significantly } \\
\text { Higher }\end{array}$ \\
\hline $\begin{array}{c}\text { VoIP } \\
\text { v6 }\end{array}$ & Very low & Very low & $\begin{array}{c}\text { Comparatively } \\
\text { lower }\end{array}$ \\
\hline
\end{tabular}

The results demonstrate that the consistency of QoS and subsequent performance are robust for VoIPv6. However, performance levels for VoIPv4 are inconsistent and that provides a significant issue for real time streaming communication applications, like VoIP or video conferencing. IPv6 based VoIP deployment performs better when taking MOS and jitter into account. For all other factors, the gradual deterioration in performance in IPv4, and thus affecting the QoS for VoIP, is undesirable. Based on these, the study indicates a higher total user experience with VoIPv6. With a very low rate of dropped traffic and insignificant experience of jitter, IPv6 is suitable for VoIP deployment. Therefore, VoIPv6 provides higher QoS than VoIPv4.

The impact of delay for real time applications like VoIP makes serious consideration of the outcome of delay related parameters important. For VoIPv4, the gradually increasing delay observed for all the parameters a potential indicator of gradual performance deterioration and lower quality of experience for users. The comparatively lower delay in VoIPv6 suggests better performance and total user experience.

\section{CONCLUSIONS AND FUTURE DEVELOPMENT}

We conclude that the transition from IPv4 to IPv6 will present significant improvements for VoIP because VoIPv6 is more efficient than VoIPv4. However, the context of an IP based communication network extends beyond the scope of the study.

The study is based on a network scenario with VoIP traffic only. In reality, an IP based network entails different kinds of traffic where VoIP, HTTP, FTP and UDP also exist. As a result, testing the efficiency of VoIP does not take into account other factors that may have an effect on the results. Therefore the study would be improved if a number of studies that contributed to the level and degree of efficiency of VoIPv4 and VoIPv6 were included.

When a study is done in a simulated environment, the efficiency of a simulation tool may be an issue because the output is directly related to the efficiency of the simulation tool and its ability to estimate and predict. OPNET is recognised as accurate in academia and industry [6], ensuring the integrity and robustness of the results obtained in the study presented in this paper. Ideally, the outcome of a simulation ought to be verified by comparing and contrasting the outcome with other similar studies. Thus the study should be considered along with other similar studies.

The study is based on specific signalling protocols and queuing mechanisms. The performance of VoIPv4 and VoIPv6 may vary if factors like signalling protocol, queuing mechanism and audio codec also vary. As a result, further study needs to address these variables. It should also be noted that the values obtained from the parameters are based on standard values. Alternative network scenarios with different configurations need to be tested, for example using H.323 or other recently developed audio codecs as a signalling protocol. The Opus audio codec is being considered as the most suitable codec for next generation IP based voice communications [23].

As part of the future study, due to the significance of various queuing mechanisms on the performance of applications like VoIP [24, 25], testing VoIP with different queuing mechanisms should be considered. The efficiency of a queuing algorithm might significantly affect the overall performance of VoIP applications. Thus, different queuing mechanisms that are to be tested for VoIP network scenarios need to be prioritized [26].

The study and analysis make it evident that further study in determining the performance for VoIP is required for both VoIPv4 and VoIPv6. While it is not possible to outline a concrete set of parameters for best performance for VoIPv4 or VoIPv6, a number of studies 
with different factors may enable optimal networking scenarios to be discovered.

\section{REFERENCES}

[1] Bi, J., Wu, J. and Leng, X. IPv4/IPv6 Transition Technologies and Univer6 Architecture. International Journal of Computer Science and Network Security, 2007. 7(1): p. 232-243.

[2] Ali, A.N.A. Comparison study between IPV4 \& IPV6. International Journal of Computer Science Issues, 2012. 9(3): p. 314-317.

[3] Dutta, C. and Singh, R. Sustainable IPv4 to IPv6 Transition. International Journal of Advanced Research in Computer Science and Software Engineering, 2012. 2 (10): p. 298-305.

[4] Reddy, P.V.P., Ali, K.M.I., Sandeep, B. and Ravi, T. Importance and Benefits of IPV6 over IPV4: A Study. International Journal of Scientific and Research Publications, 2012. 2 (12): p. 1-2.

[5] Dey, S and Shilpa, N. Issues in IPv4 to IPv6 Migration. International Journal of Computer Applications in Engineering Sciences, 2011. 1(1): p. 9-13.

[6] Karim, A. VoIP Performance Over different service Classes under Various Scheduling Techniques. Australian Journal of Basic and Applied Sciences, 2011. 5(11): p. 1416-1422.

[7] Ayokunle, O.O. Integrating Voice over Internet Protocol (VoIP) Technology as a Communication Tool on a Converged Network in Nigeria. International Journal of Information and Communication Technology Research, 2012. 2 (11): p. 829-837.

[8] Al-Ani, M.S. and Haddad, R.A.A. IPv4/IPv6 Transition. International Journal of Engineering Science and Technology, 2012.4 (12): p. 4815-4822.

[9] Abusin, A.A., Alam, M.D.J. and Abdullah, J. Testing and Analysis of VoIPv6 (Voice over Internet Protocol V6) Performance Using FreeBSD. International Journal of Communications, Network and System Sciences, 2012. 5: p. 298-302.

[10] Anouari, T. and Haqiq, A. Performance Analysis of VoIP Traffic in WiMAX using various Service Classes. International Journal of Computer Applications, 2012. 52 (20): p. 29-34.

[11] Chen, W., Wu, Q., Lin, Y. and Lo, Y. Design of SIP Application Level Gateway for IPv6 Translation. Journal of Internet Technology, 2004. 5 (2): p. 147-154.

[12] Kundu, A., Misra, I.S., Sanyal, S.K. and Bhunia, S. VoIP Performance over Broadband Wireless Networks under Static and Mobile Environments. International Journal of Wireless \& Mobile Networks, 2010. 2 (4): p. 82-93.

[13] Falk, T.H. and Chan, W. Performance Study of Objective Speech Quality Measurement for Modern Wireless VoIP Communications. EURASIP Journal on Audio, Speech, and Music Processing, 2009. Vol. 2009: p. 1-11, doi:10.1155/2009/104382.

[14] Sharma, A., Varshney, M., Singh, N.K. and Shekhar, J. Performance Evaluation of VOIP: QoS Parameters. VSRD International Journal of Computer Science \& Information Technology, 2011.1 (4): p. 210-221.

[15] Kulkarni, S., Thontadharya, H.J. and Devaraju, J.T. Performance Evaluation of VoIP in Mobile WiMAX; Simulation and Emulation studies. International Journal on Computer Science and Engineering, 2011. 3 (3): p. 1124-1130.
[16] Thaker, C., Soni, N and Patel, P. Performance Analysis and Security Provisions for VoIP Servers. International Journal of Advancements in Research \& Technology, 2013. 2 (2): p. 1-5.

[17] Ismail, M.N. Performance analysis between IPv6 and IPv4: voice over IP implementation in Campus Network. International Journal of Academic Research, 2012. 4 (5): p. 29-40.

[18] Yoo, H., Cagalaban, G.A. and Kim, S. A Study on the Connectivity of IPv6 to IPv4 Domains and Its Security Issues. International Journal of Advanced Science and Technology, 2009. 10: p. 1-10.

[19] Dawood, H.A. IPv6 Security Vulnerabilities. International Journal of Information Secirity Science, 2012. 1(4): p. 100-105.

[20] Durdagi, A. and Buldu, A. IPv4/IPv6 security and threat comparisons. Procedia Social and Behavioral Sciences, 2010. 2: p. 5285-5291.

[21] Brak, S.E., Bouhorma, M., Brak, M.E. and Bohdhir, A. Speech Quality Evaluation based Codec for VoIP over 802.11p. International Journal of Wireless \& Mobile Networks, 2013. 5(2): p. 59-69.

[22] Handley, M. Why the Internet only just works. BT Technology Journal, 2006. 24 (3): p. 119-129.

[23] Valin, J.M., Vos, K. and Terriberry, T. Definition of the Opus Audio Codec, 2012. RFC 6716.

[24] Mohammed, H.A., Ali, A.H. and Mohammed, H.J. The Affects of Different Queuing Algorithms within the Router on QoS VoIP Application using OPNET. International Journal of Computer Networks \& Communications, 2013. 5(1): p. 117-124.

[25] Nisar, K., Said, A.M. and Hasbullah, H. A Voice Priority Queue (VPQ) Fair Scheduler for the VoIP over WLANS. International Journal on Computer Science and Engineering, 3 (2): p. 506-518.

[26] Vijayakumar, M., Karthikeyani, V.and Omar, M. Implementation of Queuing Algorithm in Multipath Dynamic Routing Architecture for Effective and Secured Data Transfer in VoIP. International Journal of Engineering Trends and Technology, 2013. 4 (4): p. 12261230.

Monjur Ahmed is $\mathrm{PhD}$ student at the Service and Cloud Computing Research Lab (SCCRL), School of Computer and Mathematical Sciences (SCMS) at Auckland University of Technology. Monjur achieved his MSc in Electronics and Communications Engineering from University of Greenwich, United Kingdom, MS in Telecommunications from University of Information Technology \& Sciences, Bangladesh, and BSc (Hons) in Computing and Information Systems from London Metropolitan University, United Kingdom.

Dr Alan T Litchfield is Director of Service and Cloud Computing Research Lab (SCCRL) of School of Computer and Mathematical Sciences (SCMS) at Auckland University of Technology. Dr Litchfield is Academic Leader of the Master of Service Oriented Computing program, President of the Association for Information Systems Special Interest Group on Philosophy in Information Systems, a Member of the Institute for IT Professionals and a partner in a consulting firm that specializes in providing services to corporates and government agencies. 
Shakil Ahmed is $\mathrm{PhD}$ student at Service and Cloud Computing Research Lab (SCCRL) of School of Computer and Mathematical Sciences (SCMS) at Auckland University of Technology. Shakil achieved MSc in Mobile Computing and Communications from University of Greenwich, UK and BSc in Computer Engineering from American International University Bangladesh.

Adnan Mahmood achieved MSc in Engineering Management from University of Greenwich, United Kingdom. He works as a freelance IT consultant.
Md. Emran Hossain Meazi achieved BSc (Hons) in Computing and Information Systems from London Metropolitan University, United Kingdom and MBA from Daffodil International University, Bangladesh. $\mathrm{He}$ is a Lecturer at America Bangladesh University, Bangladesh.

How to cite this paper: Monjur Ahmed, Alan T Litchfield, Shakil Ahmed, Adnan Mahmood, Md. Emran Hossain Meazi,"VoIP Performance Analysis over IPv4 and IPv6", IJCNIS, vol.6, no.11, pp.43-48, 2014. DOI: 10.5815/ijcnis.2014.11.06 